\title{
Linear-quadratic parametrization of stabilizing controls in discrete-time 2D systems
}

\author{
P.V. Pakshin \\ Arzamas Polytechnic Institute of R.E. Alekseev Nizhny Novgorod State Technical University ,19, \\ Kalinina Street, Arzamas, 607227, Russia \\ K.Galkowski \\ Institute of Control and Computation Engineering, University of Zielona Góra, ul. Podgórna 50, 65-246 \\ Zielona Góra, Poland \\ E. Rogers
}

School of Electronics and Computer Science, University of Southampton, Southampton SO17 1BJ, UK

\begin{abstract}
This paper considers a class of linear discrete-time 2D systems in the form of repetitive processes with uncertain parameters. Using LQR theory ideas a parametric description of stabilizing controls using output feedback is developed, which leads to the development of efficient LMI-based algorithms for computation of the gain matrix. The results are extended to repetitive processes with Markovian jumps, and a numerical example is given to demonstrate the application of the algorithm developed to the synthesis of stabilizing control laws.
\end{abstract}

Keywords: uncertain 2D systems, repetitive processes, stability, control law design, Markovian jumps.

\section{Introduction}

In the fields of electrical engineering, information transmission and processing, robotics and others, dynamical processes arise that depend on two or more independent variables. The first variable can represent time, and the others can be space coordinates, numbers of iterations or trials, relating to directions of information propagation. Typical examples are processes with iterative learning control applied where in this case the time at the current learning step is the first independent variable, and the number of the learning steps, or trials, is the second. Such systems are referred to as multi-dimensional $(n \mathrm{D})$.

It is not possible to develop a comprehensive systems theory for $n \mathrm{D}$ systems as a simple extension of known results for standard (1D) systems. Even in particular cases when we are able to construct an equivalent 1D model, serious technical difficulties can arise due, for example to a dramatically increasing dimension of the problem. Also there

Email addresses: pakshin@apingtu.edu.ru (P.V. Pakshin), k.galkowski@issi.uz.zgora.pl (K.Galkowski), etar@ecs.soton.ac.uk (E. Rogers) 
are systems theoretic questions of practical relevance for $n \mathrm{D}$ systems that have no $1 \mathrm{D}$ counterparts.

An important class of two-dimensional systems are known as repetitive processes. These processes make a series of sweeps, termed passes, through a set of dynamics defined over a fixed finite duration known as the pass length $[1,2]$. On each pass, an output, known as the pass profile, is produced which acts as a forcing function on, and hence contributes to, the dynamics of the next pass profile. This, in turn, leads to the unique control problem where the output sequence of pass profiles generated can contain oscillations that increase amplitude in the pass-to-pass direction.

Physical examples of these processes include long-wall coal cutting and metal rolling operations [2] (this book gives references to the original work for these examples). In addition, applications have arisen where adopting a repetitive process setting for analysis has distinct advantages over alternatives, including classes of iterative learning control schemes [3] and iterative algorithms for solving nonlinear dynamic optimal control problems based on the maximum principle [4]. In the latter case, involving the repetitive process setting provides the basis for the development of highly reliable and efficient solution algorithms; in the former, algorithms designed using the repetitive process setting have been successfully tested experimentally on a gantry robot [3].

Other possibilities for the profitable application of repetitive process control theory have recently appeared in the modeling and control of distributed parameter systems. In particular, repetitive process state-space models arise in the modeling of spatio-temporal dynamics in, e.g., mechanical, electrical, electro-mechanical and telecommunication systems [5], [6]. Recent work in this area can be found, for example, in [7].

Attempts to stabilize these processes using 1D systems theory/algorithms fail (with the exception of a few very restrictive special cases) precisely because such an approach ignores their inherent 2D systems structure; notably, information propagation occurs from pass-to-pass and along a given pass and also the initial conditions are reset before the start of each new pass. To remove these drawbacks, a rigorous stability theory has been developed [2] based on an abstract model of the dynamics in a Banach space setting which includes a very large number of processes with linear dynamics and a constant pass length as special cases. In addition, the results of applying this theory to a range of subclasses, including the discrete-time linear repetitive processes considered here, have been reported [2]. This stability theory consists of the distinct concepts of asymptotic stability and stability along the pass, respectively.

Recognizing the unique control problem, this stability theory is of the Bounded Input Bounded Output (BIBO) form, i.e. bounded inputs are required to produce bounded sequences of pass profiles (where boundedness is defined in terms of the norm on the underlying Banach space). Asymptotic stability guarantees this property over the finite and fixed pass length, whereas stability along the pass is stronger in that it requires this property uniformly, i.e., for all possible values of the pass length (and hence it is not surprising that asymptotic stability is a necessary condition for stability along the pass).

In most practical cases, the process state-space model is not known exactly. Instead, we have (at best) nominal values for the entries in these models and to proceed we must assume that the true entries lie in some uncertainty set around the nominal one. If this set is convex, in the $1 \mathrm{D}$ case there exists a series of regular LMI-based methods to synthesize stabilizing control $[8,9]$. In this paper we extend these techniques to $2 \mathrm{D}$ systems using an affine model [8] as the uncertainty description. 
The methods provided by Letov's theory of analytical design of optimal controllers [10] (LQR theory) are one of the main synthesis tools for linear systems. The main result of this theory can be considered as a parametric description of the set of state feedback stabilizing controllers, which minimize a quadratic function. The weighting matrices of this functional play the role of parameter matrices. More recently, linear-quadratic-type parametrization has been developed for description of the set of stabilizing output feedback controllers [11]. It turns out that such LQR parametrization is suitable for application of LMI techniques. This enables the development of efficient algorithms for computation of the gain matrices. This approach has been developed in [12] for discrete-time 1D systems and extended in [13] to discrete-time 2D systems with a polytopic model of uncertainty.

In (at least) some applications of repetitive processes, it is necessary to consider the effects of failures, e.g., changes in the parameter values and/or structure of the process. One way of describing failure-prone systems is by state-space models with jumps in the parameter values and/or structure governed by a Markov chain with a finite set of states, often termed Markovian jump systems or systems with random structure, see, for example, $[14,15,17,16]$. Relevant results regarding the development of control theory for such systems, which address the issues of stability, optimal and robust control problems in the $1 \mathrm{D}$ case can be found in, for example, $[18,19,20]$ and references therein. In $[21,22]$ the results obtained for 1D Markovian jump systems are extended to investigate the problems of stabilization via state feedback $H_{\infty}$ control and $H_{\infty}$ filtering for discrete-time 2D Markovian jump systems.

The objective of this paper is to develop LQR parametrization of stabilizing static feedback control laws for linear discrete-time repetitive processes with uncertain parameters and possible failures modeled by a homogeneous Markov chain with a finite set of states. Using this parametrization, LMI-based algorithms for the computation of the stabilizing gains are derived, including robust stabilization. A numerical example is also given. The development of these new results employs stochastic quadratic stability, which includes stability along the pass as a special case.

Throughout this paper the notation $M>0$ (respectively) $M<0$ is used to denote a symmetric positive-definite (respectively negative-definite) matrix. Also $M \geq 0$ (respectively $M \leq 0$ ) is used to denote a symmetric positive (respectively negative) semi-definite matrix. The notation $|x|$ is used to denote Euclidean norm of a vector $x$, or modulus, if $x$ is real or complex number.

\section{Two-dimensional (2D) models. Basic definitions}

Two-dimensional models have been intensively studied starting from the early 1970s, generally in the fields of circuit theory, image processing and signal processing, and control. In the control area, the following models are extensively used.

- Roesser model

This model was introduced in [23] and is described by the following equations

$$
\begin{aligned}
{\left[\begin{array}{c}
x^{h}(i+1, j) \\
x^{v}(i, j+1)
\end{array}\right] } & =\left[\begin{array}{ll}
A_{11} & A_{12} \\
A_{21} & A_{22}
\end{array}\right]\left[\begin{array}{l}
x^{h}(i, j) \\
x^{v}(i, j)
\end{array}\right]+\left[\begin{array}{l}
B_{1} \\
B_{2}
\end{array}\right] u(i, j), \\
y(i, j) & =\left[\begin{array}{ll}
C_{1} & C_{2}
\end{array}\right]\left[\begin{array}{l}
x^{h}(i, j) \\
x^{v}(i, j)
\end{array}\right]+D u(i, j),
\end{aligned}
$$


where $x^{h} \in \mathbb{R}^{n^{h}}$ is the horizontal state sub-vector, $x^{v} \in \mathbb{R}^{n^{v}}$ is the vertical state sub-vector, $u \in \mathbb{R}^{r}$ is the input vector, $y \in \mathbb{R}^{m}$ is the output vector, $A_{i j}(i, j=$ $1,2), B_{i}, C_{i}(i=1,2)$, and $D$ are matrices of appropriate dimensions; the full state vector $x=\left[x^{h \mathrm{~T}} x^{v \mathrm{~T}}\right]^{\mathrm{T}}$ satisfies boundary conditions whose simplest form is

$$
x^{h}(0, j)=x_{0 j}^{h} \in \mathbb{R}^{n^{h}}, x^{v}(i, 0)=x_{i 0}^{v} \in \mathbb{R}^{n^{v}}, i, j \in Z_{+} .
$$

- Fornasini-Marchesini model

This model is defined as follows [24]

$$
\begin{aligned}
x(i+1, j+1) & =A_{1} x(i+1, j)+A_{2} x(i, j+1)+B_{1} u(i+1, j)+B_{2} u(i, j+1), \\
y(i, j) & =C x(i, j)+D u(i, j),
\end{aligned}
$$

where $x(i, j) \in \mathbb{R}^{n}$ is the state vector, $u(i, j) \in \mathbb{R}^{r}$ is the input vector, $y(i, j) \in \mathbb{R}^{m}$ is the output vector, $A_{i}(i=1,2), B, C$, and $D$ are matrices of compatible dimensions. The simplest form of boundary conditions are

$$
x(0, j)=x_{0 j} \in \mathbb{R}^{n}, x(i, 0)=x_{i 0} \in \mathbb{R}^{n} i, j \in Z_{+} .
$$

- Linear repetitive processes

This model is formally similar to 2D Roesser model and is defined as follows [2].

$$
\begin{aligned}
x_{k+1}(p+1) & =A x_{k+1}(p)+B_{0} y_{k}(p)+B u_{k+1}(p), \\
y_{k+1}(p) & =C x_{k+1}(p)+D_{0} y_{k}(p)+D u_{k+1}(p),
\end{aligned}
$$

where $x_{k}(p) \in \mathbb{R}^{n}$ is the state vector, $u_{k}(p) \in \mathbb{R}^{r}$ is the input vector, $y_{k}(p) \in \mathbb{R}^{m}$ is the output (pass profile) vector, $k \in Z_{+}$is the current pass number, $0 \leq p \leq \alpha-1 \in$ $Z_{+}$is the discrete position on the current pass $(\alpha<\infty), A, B, B_{0}, C, D$, and $D_{0}$ are matrices of compatible dimensions; the simplest form of the boundary conditions is

$$
x_{k+1}(0)=d_{k+1} \in \mathbb{R}^{n}, k \geq 0, y_{0}(p)=f(p) \in \mathbb{R}^{m},
$$

where $f(p)$ is known vector function of $p$.

Many difficulties appear arise in the systems related analysis for even the linear models given above.the dynamics. In the case of repetitive processes the stability theory for linear constant pass length examples is based on an abstract model in a Banach space setting which includes many such spaces as special cases. Let $E_{\alpha}$ be a Banach space and $W_{\alpha}$ a linear subspace of $E_{\alpha}$, also denote the norm on $E_{\alpha}$ as $\|\cdot\|$. Then this model is given by

$$
y_{k+1}=L_{\alpha} y_{k}+b_{k+1}, k \geq 0,
$$

where $y_{k} \in E_{\alpha}$ is the pass profile on pass $k, L_{\alpha}$ is a bounded linear operator mapping $E_{\alpha}$ into itself, and $b_{k+1} \in W_{\alpha}$ represent terms that enter on pass $k+1$. In the special case of an example described by (1) and (2), take $E_{\alpha}=l_{2}^{m}$ of the $m$-dimensional vectors given on the length $\left[\begin{array}{ll}0 & \alpha\end{array}\right]$. Then

$$
\begin{aligned}
L_{\alpha} y(p) & =\sum_{i=0}^{p-1} C A^{p-1-i} B_{0} y(i)+D_{0} y(p), \\
b_{k+1} & =C A^{p} d_{k+1}+\sum_{i=0}^{p-1} C A^{p-1-i} B u_{k+1}(i)+D u_{k+1}(p), 1 \leq p \leq \alpha, k \geq 0 .
\end{aligned}
$$


Definition 1 ([2, p. 41]). A linear repetitive process described by (4) is said to be asymptotically stable if there exists a real scalar $\delta>0$ such that, given any initial pass profile $y_{0}$ and any strongly convergent as $k \rightarrow \infty$ disturbance sequence $\left\{b_{k}\right\}_{k \geq 1}$, the sequence $\left\{y_{k}\right\}_{k \geq 1}$ generated by the perturbed process

$$
y_{k+1}=\left(L_{\alpha}+\gamma\right) y_{k}+b_{k+1}, k \geq 0
$$

converges strongly to a limit profile $y_{\infty}$ as $k \rightarrow \infty$ whenever $\|\gamma\|<\delta$.

Asymptotically stable systems have the convergence property given by the following lemma.

Lemma 1 ([2, p. 56]). Suppose that the linear repetitive process described by (4) is asymptotically stable and the constant disturbance sequence $b_{k+1}=b_{\infty}, k \geq 0$ generates the limit profile $y_{\infty}$. Then there exist real scalars $M_{\alpha}$ and $\lambda_{\alpha} \in(0,1)$ such that

$$
\left\|y_{k}-y_{\infty}\right\| \leq M_{\alpha} \lambda_{\alpha}^{k}\left(\left\|y_{0}\right\|+\frac{\left\|b_{\infty}\right\|}{1-\lambda_{\alpha}}\right), k \geq 0
$$

Actually, the last result states that under asymptotic stability the output sequence $\left\{y_{k}\right\}_{k \geq 1}$ approaches the resulting limit profile $y_{\infty}$ at a geometric rate governed by the scalar $\lambda_{\alpha}$. Given that the pass length is finite, it is possible that the limit profile of an asymptotically stable process can have unacceptable along the pass dynamics. One way of ensuring that this does not happen is to use stability along the pass expressed in terms of finite bounds on the scalars $M_{\alpha}$ and $\lambda_{\alpha}$ as $\alpha \rightarrow \infty$. This property requires that the rate of approach of the output sequence to the limit profile has a guaranteed geometric upper bound independent of the pass length.

Definition 2 ([2, p. 57]). A linear repetitive process described by (4) is said to be stable along the pass if there exist finite real scalars $M_{\infty}$ and $\lambda_{\infty} \in(0,1)$ (independent of $\alpha$ ) such that for each constant disturbance sequence $b_{k+1}=b_{\infty}, k \geq 0$ the corresponding output sequence satisfies

$$
\left\|y_{k}-y_{\infty}\right\| \leq M_{\infty} \lambda_{\infty}^{k}\left(\left\|y_{0}\right\|+\frac{\left\|b_{\infty}\right\|}{1-\lambda_{\infty}}\right), k \geq 0 .
$$

Theorem 1 ([2, p. 64]). Suppose that the pair $\left(A, B_{0}\right)$ is controllable and the pair $(C, A)$ is observable. Then the linear repetitive process defined by (1), (2) is stable along the pass if, and only if, its characteristic polynomial

$$
\mathcal{C}\left(z_{1}, z_{2}\right)=\operatorname{det}\left[\begin{array}{cc}
I_{n}-z_{1} A & -z_{1} B_{0} \\
-z_{2} C & I_{m}-z_{2} D_{0}
\end{array}\right]
$$

satisfies

$$
\mathcal{C}\left(z_{1}, z_{2}\right) \neq 0 \forall\left(z_{1}, z_{2}\right):\left|z_{1}\right| \leq 1,\left|z_{2}\right| \leq 1
$$

The checking of this last result for a given example can prove problematic and an alternative is to use Lyapunov's method and obtain the following results 
Theorem 2 ([2, p. 102]). A discrete linear repetitive process defined by (1), (2) is stable along the pass if there exists a symmetric positive definite matrix $P=\operatorname{diag}\left[P_{1} P_{2}\right]$, satisfying the Lyapunov inequality

$$
\bar{A}^{T} P \bar{A}-P<0
$$

where $\bar{A}=\left[\begin{array}{cc}A & B_{0} \\ C & D_{0}\end{array}\right]$.

In this result the solution of the Lyapunov inequality must have a special block-diagonal form and this imposes additional constraints. The Lyapunov method leads directly to control law design algorithms whereas this is more difficult starting from the condition of Theorem 1.

\section{Linear-quadratic parametrization of stabilizing controls of uncertain repet- itive processes}

\subsection{Problem statement and preliminary results}

Consider uncertain discrete linear repetitive processes described by the following statespace model

$$
\begin{aligned}
x_{k+1}(p+1) & =A(\gamma) x_{k+1}(p)+B(\gamma) u_{k+1}(p)+B_{0}(\gamma) y_{k}(p) \\
y_{k+1}(p) & =C(\gamma) x_{k+1}(p)+D(\gamma) u_{k+1}(p)+D_{0}(\gamma) y_{k}(p)
\end{aligned}
$$

where $x_{k}(p)$ is the $n \times 1$ current pass state vector, $y_{k}(p)$ is the $m \times 1$ pass profile vector, $u_{k}(p)$ is the $r \times 1$ is current pass input (control) vector, the boundary conditions are specified in the form (3); the matrices $A(\gamma), B(\gamma), B_{0}(\gamma), C(\gamma), D(\gamma), D_{0}(\gamma)$ are affine functions of uncertain parameters vector $\gamma=\left[\gamma_{1} \ldots \gamma_{N}\right]^{T}$, i.e.,

$$
A(\gamma)=A_{0}+\sum_{i=1}^{N} \gamma_{i} A_{i}, B(\gamma)=B_{0}+\sum_{i=1}^{N} \gamma_{i} B_{i}, B_{0}(\gamma)=B_{00}+\sum_{i=1}^{N} \gamma_{i} B_{0 i}
$$

and so on. In addition, $\gamma_{i},(i=1, \ldots, N)$ are assumed to be bounded in an interval including zero

$$
\underline{\gamma}_{i} \leq \gamma_{i} \leq \bar{\gamma}_{i}: \quad \underline{\gamma}_{i} \leq 0, \quad \bar{\gamma}_{i} \geq 0, i=1, \ldots, N
$$

The set of uncertainties vectors $\gamma=\left[\gamma_{1} \ldots \gamma_{N}\right]^{T}$ satisfying inequalities (9) is denoted by $\Gamma$. The finite set of extremal values or vertices is defined by

$$
\Gamma_{v}=\left\{\gamma=\left[\gamma_{1} \ldots \gamma_{N}\right]^{T}: \gamma_{i} \in\left\{\underline{\gamma}_{i}, \bar{\gamma}_{i}\right\}, i=1, \ldots, N\right\}
$$

Consider static output feedback control laws of the form

$$
u_{k+1}(p)=-F_{1} \xi_{k+1}(p)-F_{2} \eta_{k}(p),
$$

where $\xi_{k+1}(p)=\Theta_{1} x_{k+1}(p)$ and $\eta_{k}(p)=\Theta_{2} y_{k}(p)$ are measurable outputs, and $\Theta_{1}$ and $\Theta_{2}$ are rectangular full-row rank matrices of compatible dimensions. The form of this control means we also address the practically relevant issue that all of the repetitive process variables are not directly measured. The particular choice of $\Theta_{1}=I$ and $\Theta_{2}=I$ recovers the ideal case of this control law considered in [2]. The problem addressed next in this 
paper is to find $F_{1}$ and $F_{2}$ such that the control law (11) stabilizes a process described by (8)-(11) in the along the pass sense.

Consider the function

$$
V(k, p)=x_{k+1}^{T}(p) W_{1} x_{k+1}(p)+y_{k}^{T}(p) W_{2} y_{k}(p)
$$

where $W_{1}=W_{1}^{T}>0$ and $W_{2}=W_{2}^{T}>0$, with associated increment along the trajectories of $(8)$

$$
\begin{array}{r}
\Delta V(k, p)=x_{k+1}^{T}(p+1) W_{1} x_{k+1}(p+1)-x_{k+1}^{T}(p) W_{1} x_{k+1}(p) \\
+y_{k+1}^{T}(p) W_{2} y_{k+1}(p)-y_{k}^{T}(p) W_{2} y_{k}(p) .
\end{array}
$$

Now we have the following result.

Lemma 2 ([2, 13]). A discrete linear repetitive process described by (8)-(10)is stable along the pass if there exists a function (12) with associated increment along the trajectories of (8) satisfying

$$
\Delta V(k, p)<0
$$

for all $k \geq 0,0 \leq p \leq \alpha-1$.

Introduce the matrices

$$
\begin{aligned}
& \bar{A}(\gamma)=\left[\begin{array}{ll}
A(\gamma) & B_{0}(\gamma) \\
C(\gamma) & D_{0}(\gamma)
\end{array}\right], \bar{B}(\gamma)=\left[\begin{array}{c}
B(\gamma) \\
D(\gamma)
\end{array}\right], \gamma \in \Gamma_{v} \\
& F=\left[\begin{array}{ll}
F_{1}, & F_{2}
\end{array}\right], \Theta=\operatorname{diag}\left[\Theta_{1}, \Theta_{2}\right] .
\end{aligned}
$$

Since the uncertainty has an affine model structure, the condition (14) is reduced to checking $2^{N}$ Lyapunov inequalities. The following result gives a sufficient condition for stability along the pass.

Theorem 3 ([2, 13]). A discrete linear repetitive process described by (8)-(10) is stable along the pass if there exist matrices $W_{1}=W_{1}^{T}>0 \quad W_{2}=W_{2}^{T}>0$ such that the system of Lyapunov inequalities

$$
\bar{A}(\gamma)^{T} W \bar{A}(\gamma)-W<0, \quad \gamma \in \Gamma_{v}
$$

has a positive definite solution $W=\operatorname{diag}\left[W_{1} W_{2}\right]$.

\subsection{LQR-type parametrization}

Suppose that the control law (11) is applied to a process described by (8). Then applying Theorem 3 to the resulting controlled process yields stability along the pass if there exist a positive definite matrix $W=\operatorname{diag}\left[W_{1}, W_{2}\right]$ and a matrix $F=\left[\begin{array}{ll}F_{1} & F_{2}\end{array}\right]$ such that

$$
(\bar{A}(\gamma)-\bar{B}(\gamma) F \Theta)^{T} W(\bar{A}(\gamma)-\bar{B}(\gamma) F \Theta)-W<0, \quad \gamma \in \Gamma_{v}
$$

The following theorem gives a parametric description of all matrices $F$ satisfying (17) and is an extension of the LQR-type parametrization idea $[11,12]$ to discrete linear repetitive processes. 
Theorem 4. There exists a matrix F satisfying (17) if, and only if, there exist matrices $Q(\gamma)=Q(\gamma)^{T} \geq 0, R(\gamma)=R(\gamma)^{T}>0$ and $L(\gamma)\left(\gamma \in \Gamma_{v}\right)$ such that

$$
\begin{array}{r}
F \Theta=\left[\bar{B}(\gamma)^{T} W \bar{B}(\gamma)+R(\gamma)\right]^{-1}\left[\bar{B}(\gamma)^{T} W \bar{A}(\gamma)+L(\gamma)\right], \gamma \in \Gamma_{v} \\
{\left[\bar{B}(\delta)^{T} W \bar{B}(\delta)+R(\delta)\right]^{-1}\left[\bar{B}(\delta)^{T} W \bar{A}(\delta)+L(\delta)\right]=} \\
=\left[\bar{B}(\gamma)^{T} W \bar{B}(\gamma)+R(\gamma)\right]^{-1}\left[\bar{B}(\gamma)^{T} W \bar{A}(\gamma)+L(\gamma)\right], \delta, \gamma \in \Gamma_{v}
\end{array}
$$

where $W=\operatorname{diag}\left[W_{1} W_{2}\right]$ is a solution of

$$
\begin{aligned}
\bar{A}(\gamma)^{T} W \bar{A}(\gamma)- & W-\bar{A}(\gamma)^{T} W \bar{B}(\gamma)\left[\bar{B}(\gamma)^{T} W \bar{B}(\gamma)+R(\gamma)\right]^{-1} \bar{B}(\gamma)^{T} W \bar{A}(\gamma) \\
& +L(\gamma)^{T}\left[\bar{B}(\gamma)^{T} W \bar{B}(\gamma)+R(\gamma)\right]^{-1} L(\gamma)+Q(\gamma)<0, \gamma \in \Gamma_{v}
\end{aligned}
$$

The proof of this result is given in Appendix A.

In this result the parameter matrices $Q(\gamma) \geq 0$ and $R(\gamma)>0, \gamma \in \Gamma_{v}$ play the same roles as the state and control weighting matrices in the $1 \mathrm{D}$ discrete-time linear systems LQR problem. Theorem 4 gives no direct technique to calculate a stabilizing $F$. However, in contrast to direct application of the system of inequalities (17), the parameter matrices provide a certain degree of freedom, and this is exploited next to develop sufficient conditions that lead to Linear Matrix Inequality (LMI)-based algorithms for this task.

To begin suppose that for some $\mu(\gamma)>0$

$$
\begin{array}{r}
{\left[\begin{array}{cc}
\mu(\gamma) Q(\gamma) & L(\gamma)^{T} \\
L(\gamma) & \bar{B}(\gamma)^{T} W \bar{B}(\gamma)+R(\gamma)
\end{array}\right]>0} \\
\bar{A}(\gamma)^{T} W \bar{A}(\gamma)-W-\bar{A}(\gamma)^{T} W \bar{B}(\gamma)\left[\bar{B}(\gamma)^{T} W \bar{B}(\gamma)\right. \\
+R(\gamma)]^{-1} \bar{B}(\gamma)^{T} W \bar{A}(\gamma)+Q_{\mu}(\gamma) \leq 0, \gamma \in \Gamma_{v}
\end{array}
$$

where $Q_{\mu}(\gamma)=[1+\mu(\gamma)] Q(\gamma)$ and it follows immediately that (20) holds. In addition, (18) is solvable with respect to $F$, if and only if [25]

$$
\left[\bar{B}(\gamma)^{T} W \bar{A}(\gamma)+L(\gamma)\right]\left[I-\Theta^{+} \Theta\right]=0, \gamma \in \Gamma_{v}
$$

where the superscript + denotes the Moore-Penrose inverse. The relations (21), (22), and (23) are convex, but cannot be directly solved since (22) is nonlinear with respect to initial unknown variables. To find a stabilizing $F$, the fact used [26] is that the solution of the LMI optimization problem

$$
\max \operatorname{tr} W(\gamma)
$$

under the constraints

$$
\begin{aligned}
& {\left[\begin{array}{cc}
\bar{A}(\gamma)^{T} W(\gamma) \bar{A}(\gamma)-W(\gamma)+Q_{\mu}(\gamma) & \bar{A}(\gamma)^{T} W(\gamma) \bar{B}(\gamma) \\
\left(\bar{A}(\gamma)^{T} W(\gamma) \bar{B}(\gamma)\right)^{T} & \bar{B}(\gamma)^{T} W(\gamma) \bar{B}(\gamma)+R(\gamma)
\end{array}\right] \geq 0} \\
& W(\gamma)=W(\gamma)^{T}>0, \gamma \in \Gamma_{v},
\end{aligned}
$$

coincides with the positive definite solution $W(\gamma)=W(\gamma)^{T}$ of the Riccati equation

$$
\begin{array}{r}
\bar{A}(\gamma)^{T} W(\gamma) \bar{A}(\gamma)-W(\gamma)-\bar{A}(\gamma)^{T} W(\gamma) \bar{B}(\gamma)\left[\bar{B}_{r}^{T} W(\gamma) \bar{B}(\gamma)\right. \\
+R(\gamma)]^{-1} \bar{B}(\gamma)^{T} W(\gamma) \bar{A}(\gamma)+Q_{\mu}(\gamma)=0, \gamma \in \Gamma_{v}
\end{array}
$$


The problem considered here has the also has the constraints (21) and (23). Moreover, the matrix $W$ is diagonal and hence the algorithm below is based on finding a solution of the LME/LMI problem (19), (21) and

$$
\left[\begin{array}{cc}
\bar{A}(\gamma)^{T} W \bar{A}(\gamma)-W+Q_{\mu i} & \bar{A}(\gamma)^{T} W \bar{B}(\gamma) \\
\left(\bar{A}(\gamma)^{T} W \bar{B}(\gamma)\right)^{T} & \bar{B}(\gamma)^{T} W \bar{B}(\gamma)+R(\gamma)
\end{array}\right] \geq 0, \gamma \in \Gamma_{v}
$$

i.e., as close as possible to the block diagonal positive definite solution $W=\operatorname{diag}\left[\begin{array}{ll}W_{1} & W_{2}\end{array}\right]$ of the system of Riccati equations

$$
\begin{array}{r}
\bar{A}(\gamma)^{T} W \bar{A}(\gamma)-W-\bar{A}(\gamma)^{T} W \bar{B}(\gamma)\left[\bar{B}(\gamma)^{T} W \bar{B}(\gamma)\right. \\
+R(\gamma)]^{-1} \bar{B}(\gamma)^{T} W \bar{A}(\gamma)+Q_{\mu i}=0, \gamma \in \Gamma_{v}
\end{array}
$$

This method will be fruitful if the matrices $Q(\gamma)$ and $R(\gamma)$ are chosen to provide a sufficient stability margin for the closed-loop system. An LMI-based algorithm for computing the stabilizing control law matrices includes the following steps.

\section{Algorithm 1.}

1. Select the matrices $Q(\gamma)$ and $R(\gamma)$ based on $L Q R$ reasoning.

2. Solve the LMI/LME optimization problem

$$
\operatorname{tr} W \rightarrow \max
$$

under the constraints (26).

3. If the problem of the previous step is feasible, compute the stabilizing control law matrix F using

$$
F=\left[\bar{B}(\gamma)^{T} W \bar{B}(\gamma)+R(\gamma)\right]^{-1}\left[\bar{B}(\gamma)^{T} W \bar{A}(\gamma)+L(\gamma)\right] \Theta^{+}
$$

where $\gamma$ is selected arbitrarily from the set $\Gamma_{v}$.

4. If the $L M I$

$$
(\bar{A}(\gamma)-\bar{B}(\gamma) F \Theta)^{T} S(\bar{A}(\gamma)-\bar{B}(\gamma) F \Theta)-S<0, \gamma \in \Gamma_{v}
$$

is feasible with respect to $S=\operatorname{diag}\left[S_{1}, S_{2}\right]>0$, then $F$ is stabilizing control law matrix.

This algorithm is the discrete linear repetitive process counterpart of that developed in [12] for 1D discrete-time linear systems.

\section{Linear-quadratic parametrization of stabilizing controls of repetitive pro- cesses with Markovian jumps}

In this section we consider the case when the structure of repetitive process is subject to random failures. In this case the process is described by the following state-space model

$$
\begin{aligned}
x_{k+1, p+1} & =A\left(\rho_{k, p}\right) x_{k+1, p}+B\left(\rho_{k, p}\right) u_{k+1, p}+B_{0}\left(\rho_{k, p}\right) y_{k, p}, \\
y_{k+1, p} & =C\left(\rho_{k, p}\right) x_{k+1, p}+D\left(\rho_{k, p}\right) u_{k+1, p}+D_{0}\left(\rho_{k, p}\right) y_{k, p},
\end{aligned}
$$

where $\rho_{k, p}$ is homogeneous Markov process with finite set of states $\mathbb{N}=\{1, \ldots, \nu\}$ and with transition probabilities

$$
\begin{aligned}
& \mathrm{P}\left[\rho_{k, p+1}=j \mid \rho_{k, p}=i\right]=\pi_{i j}, \\
& \mathrm{P}\left[\rho_{k+1, p}=j \mid \rho_{k, p}=i\right]=\omega_{i j} .
\end{aligned}
$$


Note that from this point onwards subscripts for independent variables to make notation compact. The remainder of the notation is identical to that of (1), (2) or (8).

Consider an output feedback control law of the form

$$
u_{k+1, p}=\beta\left(\rho_{k, p}\right)\left[\varphi\left(x_{k+1, p}\right)+\psi\left(y_{k, p}\right)\right], \varphi(0)=0, \psi(0)=0
$$

and introduce the following definition of asymptotic stability in the mean square for the system considered in this section.

Definition 3. A $2 D$ system of the form (30) with output feedback control law (32) applied is said to be asymptotically stable in the mean square sense if for all boundary conditions of the form (3) satisfying the inequality

$$
\mathrm{E}\left[\sum_{i=0}^{\infty}\left|d_{i+1}\right|^{2}+\left|f_{i}\right|^{2}\right]<\infty,
$$

the following holds

$$
\mathrm{E}\left[\left|x_{k+1, p}\right|^{2}+\left|y_{k, p}\right|^{2}\right] \rightarrow 0
$$

as $i+j \rightarrow \infty$, where $\mathrm{E}$ denotes the expectation operator.

Consider the candidate stochastic Lyapunov function

$$
V(x, y, r)=V_{1}(x, r)+V_{2}(y, r), V_{1}(0, r)=0, V_{2}(0, r)=0, x \in \mathbb{R}^{n}, y \in \mathbb{R}^{m}, r \in \mathbb{N}
$$

with associated increment along the trajectories of the system (30) with control law (32) applied

$$
\begin{aligned}
\Delta V(x, y, r) & =\mathrm{E}\left[V_{1}\left(x_{k+1, p+1}, \rho_{k, p+1}\right)+V_{2}\left(y_{k+1, p}, \rho_{k+1, p}\right)-\right. \\
& \left.-V_{1}\left(x_{k+1, p}, \rho_{k, p}\right)-V_{2}\left(y_{k, p}, \rho_{k, p}\right) \mid x_{k+1, p}=x, y_{k, p}=y, \rho_{k, p}=r\right] .
\end{aligned}
$$

Theorem 5. Suppose there exist positive constants $c_{1}, c_{2}, c_{3}$ such that the Lyapunov function (34) and its associated increment (35) along the trajectories of the system (30) with control law (32) applied satisfy

$$
\begin{aligned}
c_{1}\left(\left|x_{k+1, p}\right|^{2}+\left|y_{k, p}\right|^{2}\right) \leq V\left(x_{k, p}, y_{k, p}, \rho_{k, p}\right) & \leq c_{2}\left(\left|x_{k+1, p}\right|^{2}+\left|y_{k, p}\right|^{2}\right), \\
\Delta V\left(x_{k, p}, y_{k, p}, \rho_{k, p}\right) & \leq-c_{3}\left(\left|x_{k+1, p}\right|^{2}+\left|y_{k, p}\right|^{2}\right),
\end{aligned}
$$

Then this system is asymptotically stable in the mean square sense.

The proof of this result is given in Appendix B.

Consider the case when the control law has the form of linear feedback which is switched synchronously with jumps of a Markov process:

$$
u_{k+1, p}=-F_{1}(r) \xi_{k+1, p}-F_{2}(r) \eta_{k, p}, \quad \rho_{k, p}=r,
$$

where $\xi_{k+1, p}=\Theta_{1}\left(r_{k, p}\right) x_{k+1, p}, \eta_{k, p}=\Theta_{2}\left(r_{k, p}\right) y_{k, p}$ denote measurable outputs, and $\Theta_{1}(r)$ and $\Theta_{2}(r)(r \in \mathbb{N})$ are rectangular full-row rank matrices of compatible dimensions. Also choose the function $V(x, y, r)$ in the quadratic form

$$
\begin{array}{r}
V(x, y, r)=x^{T} W_{1}(r) x+y^{T} W_{2}(r) y \\
W_{1}(r)=W_{1}^{T}(r)>0, W_{2}(r)=W_{2}^{T}(r)>0, r \in \mathbb{N} .
\end{array}
$$


Introduce the notation

$$
\begin{array}{r}
\bar{A}(r)=\left[\begin{array}{cc}
A(r) & B_{0}(r) \\
C(r) & D_{0}(r)
\end{array}\right], \bar{B}(r)=\left[\begin{array}{c}
B(r) \\
D(r)
\end{array}\right], \\
F(r)=\left[F_{1}(r), \quad F_{2}(r)\right], \Theta(r)=\operatorname{diag}\left[\Theta_{1}(r), \quad \Theta_{2}(r)\right] r \in \mathbb{N} .
\end{array}
$$

Then the conditions of Theorem 5 hold if the system of matrix inequalities

$$
\left.\left(\bar{A}(r)-\bar{B}_{r} F_{r} \Theta_{r}\right)^{T} \bar{W}(r) \bar{A}(r)-\bar{B}_{r} F_{r} \Theta_{r}\right)-W(r)<0, r \in \mathbb{N},
$$

where $\bar{W}(r)=\operatorname{diag}\left[\sum_{j=1}^{\nu} \pi_{r j} W_{1}(j) \sum_{j=1}^{\nu} \omega_{r j} W_{2}(j)\right], W(r)=\operatorname{diag}\left[W_{1}(r) W_{2}(r)\right]$, is solvable with respect to matrices $W_{1}(r)=W_{1}^{T}(r)>0, W_{2}(r)=W_{2}^{T}(r)>0,(r \in \mathbb{N})$.

The following theorem gives a parametric description of all matrices $F(r)$ satisfying (40).

Theorem 6. There exists a matrix $F(r)$ satisfying (40) if, and only if, there exist matrices $Q(r)=Q(r)^{T} \geq 0, R(r)=R(r)^{T}>0$ and $L(r)(r \in \mathbb{N})$ such that

$$
F(r) \Theta(r)=\left[\bar{B}(r)^{T} \bar{W}(r) \bar{B}(r)+R(r)\right]^{-1}\left[\bar{B}(r)^{T} \bar{W}_{r} \bar{A}_{r}+L_{r}\right], r \in \mathbb{N},
$$

where $W=\operatorname{diag}\left[W_{1} W_{2}\right]$ is a solution of

$$
\begin{array}{r}
\bar{A}(r)^{T} \bar{W}(r) \bar{A}(r)-W(r)- \\
-\bar{A}(\gamma)^{T} \bar{W}(r) \bar{B}(r)\left[\bar{B}(r)^{T} \bar{W}(r) \bar{B}(r)+R(r)\right]^{-1} \bar{B}(r)^{T} \bar{W}(r) \bar{A}(r)+ \\
+L(r)^{T}\left[\bar{B}(r)^{T} \bar{W}(r) \bar{B}(r)+R(r)\right]^{-1} L(r)+Q(r)<0, r \in \mathbb{N}
\end{array}
$$

and $\bar{W}(r)=\operatorname{diag}\left[\sum_{j=1}^{\nu} W_{1}(j) \pi_{r j} \sum_{j=1}^{\nu} W_{2}(j) \omega_{r j}\right], r \in \mathbb{N}$.

The proof of this result is omitted as it follows by routine changes to that for Theorem 4 .

To find the gain matrix $F(r)$ we use an idea based on the general result in [26], which states that the solution of the optimization problem

$$
\max \operatorname{tr} \sum_{r=1}^{N} W(r)
$$

under the constraints

$$
\begin{gathered}
{\left[\begin{array}{cc}
\bar{A}(r)^{T} \bar{W}(r) \bar{A}(r)-W(r)+Q(r) & \bar{A}(r)^{T} \bar{W}(r) \bar{B}(r) \\
\left(\bar{A}(r)^{T} \bar{W}(r) \bar{B}(r)\right)^{T} & \bar{B}(r)^{T} \bar{W}(r) \bar{B}(r)+R(r)
\end{array}\right] \geq 0,} \\
W(r)=W(r)_{i}^{T}>0, \bar{W}(r)=\sum_{j=1}^{\nu} W_{j} \pi_{r j}, \pi_{r j} \geq 0, \sum_{j=1}^{\nu} \pi_{r j}=1, r \in \mathbb{N},
\end{gathered}
$$

coincides with the positive definite solution $W(r)=W(r)^{T}$ of the system of Riccati equations

$$
\begin{array}{r}
\bar{A}(r)^{T} \bar{W}(r) \bar{A}(r)-W(r)-\bar{A}(r)^{T} \bar{W}(r) \bar{B}(r)\left[\bar{B}(r)^{T} \bar{W}(r) \bar{B}(r)\right. \\
+R(r)]^{-1} \bar{B}(r)^{T} \bar{W}(r) \bar{A}(r)+Q(r)=0, r \in \mathbb{N} .
\end{array}
$$

Proceeding with similar reasons to those in the previous section, the following is an algorithm for computing the gain matrix. 


\section{Algorithm 2.}

1. Select the matrices $Q(r)$ and $R(r)(r \in \mathbb{N})$ based on $L Q R$ reasoning.

2. Solve the LMI/LME optimization problem

$$
\max \operatorname{tr} \sum_{r=1}^{\nu} W(r)
$$

under the following constraints in the form of linear matrix equations and inequalities

$$
\begin{array}{r}
{\left[\bar{B}(r)^{T} \bar{W}(r) \bar{A}(r)+L(r)\right]\left[I-\Theta(r)^{+} \Theta(r)\right]=0} \\
{\left[\begin{array}{rr}
\bar{A}(r)^{T} \bar{W}(r) \bar{A}(r)-W(r)+(1+\mu(r)) Q(r) & \bar{A}(r)^{T} \bar{W}(r) \bar{B}(r) \\
\left(\bar{A}(r)^{T} \bar{W}(r) \bar{B}(r)\right)^{T} & \bar{B}(r)^{T} \bar{W}(r) \bar{B}(r)+R(r)
\end{array}\right] \geq 0} \\
{\left[\begin{array}{cc}
\mu(r) Q(r) & L(r)^{T} \\
L(r) & \bar{B}(r)^{T} \bar{W}(r) \bar{B}(r)+R(r)
\end{array}\right]>0} \\
\bar{W}(r)=\operatorname{diag}\left[\begin{array}{ll}
\sum_{j=1}^{\nu} W_{1}(j) \pi_{r j} & \sum_{j=1}^{\nu} W_{2}(j) \omega_{r j}
\end{array}\right], r \in \mathbb{N} .
\end{array}
$$

3. If the problem of the previous step is feasible, compute the gain matrix as

$$
F(r)=\left[\bar{B}^{T} \bar{W}(r) \bar{B}(r)+R(r)\right]^{-1}\left[\bar{B}(r)^{T} \bar{W}(r) \bar{A}(r)+L(r)\right] \Theta(r)^{+}, r \in \mathbb{N} .
$$

4. If the system of linear matrix inequalities

$$
\begin{array}{r}
(\bar{A}(r)-\bar{B}(r) F(r) \Theta(r))^{T} \bar{S}(r)(\bar{A}(r)-\bar{B}(r) F(r) \Theta(r)) \\
-S(r)<0, r \in \mathbb{N}
\end{array}
$$

where $S(r)=\operatorname{diag}\left[S_{1 r}, \quad S_{2 r}\right], \quad \bar{S}(r)=\operatorname{diag}\left[\sum_{j=1}^{\nu} S_{1}(j) \pi_{r j} \sum_{j=1}^{\nu} S_{2}(j) \omega_{r j}\right]$, is feasible with respect to $S(r)=S(r)^{T}>0$, then $F(r)$ is the gain matrix of the stabilizing control law.

\section{Numerical example}

Consider an unstable discrete repetitive process described by (30) for which a stabilizing control in the form of (38) has been obtained. Suppose the Markov process does not depend on the variable $k$, i.e., $\rho_{k, p}=\rho_{p}$ and

$$
\begin{gathered}
\mathrm{P}\left[\rho_{p+1}=j \mid \rho_{p}=i\right]=\pi_{i j}, \omega_{i j}=0, i \neq j, \omega_{i i}=1 i, j \in \mathbb{N} \\
\mathbb{N}=\{1,2\}, \quad \Pi=\left[\begin{array}{cc}
0.5 & 0.5 \\
0.1 & 0.9
\end{array}\right]
\end{gathered}
$$

Then

$$
\begin{gathered}
A(1)=\left[\begin{array}{cc}
-0.72 & 0.08 \\
0.0 & 1.7
\end{array}\right], A(2)=\left[\begin{array}{cc}
-0.37 & 0.51 \\
-0.13 & -0.16
\end{array}\right], \\
B_{0}(1)=\left[\begin{array}{cc}
0.0 & -0.72 \\
0.0 & 0.0
\end{array}\right], B_{0}(2)=\left[\begin{array}{ccc}
-0.49 & -0.7 \\
0.0 & 0.0
\end{array}\right], \\
B(1)=\left[\begin{array}{cccc}
1.6 & 0.41 & -0.27 & 1.42 \\
0.54 & -2.51 & 1.76 & 1.03
\end{array}\right],
\end{gathered}
$$




$$
\begin{aligned}
& B(2)=\left[\begin{array}{cccc}
-1.55 & 1.38 & -0.11 & 1.98 \\
0.8 & 0.66 & 2.29 & 0.38
\end{array}\right], \\
& C(1)=\left[\begin{array}{ll}
0.32 & 0.0 \\
0.29 & 0.0
\end{array}\right], C(2)=\left[\begin{array}{cc}
0.0 & 0.0 \\
0.0 & 0.97
\end{array}\right] \text {, } \\
& D_{0}(1)=\left[\begin{array}{cc}
0.1 & 0.6 \\
0.18 & -0.38
\end{array}\right], D_{0}(2)=\left[\begin{array}{cc}
1.12 & -0.05 \\
0.78 & -0.01
\end{array}\right] \text {, } \\
& D(1)=\left[\begin{array}{cccc}
0.12 & -0.48 & 0.29 & 0.56 \\
2.67 & -2.87 & -1.49 & -2.3
\end{array}\right], \\
& D(2)=\left[\begin{array}{cccc}
0.0 & 0.0 & -2.89 & -1.78 \\
-2.96 & -0.22 & 0.81 & 0.21
\end{array}\right] \text {, } \\
& \Theta(1)=\left[\begin{array}{llll}
1 & 0 & 0 & 0 \\
0 & 1 & 0 & 0 \\
0 & 0 & 0 & 1
\end{array}\right], \Theta(2)=\left[\begin{array}{llll}
1 & 0 & 0 & 0 \\
0 & 0 & 1 & 0
\end{array}\right] .
\end{aligned}
$$

In addition, set $Q(r)=I R(r),=I$ and $\mu(r)=1, r=1,2$. The eigenvalues of the matrix $\bar{A}(r)$ in the first and second modes are $\Lambda(1)=[0.1782-0.5891+0.3568 i-0.5891-$ $0.3568 i 1.7]$ and $\Lambda(2)=[-0.3956+0.4239 i-0.3956-0.4239 i 0.29011 .0811]$, respectively. Applying Algorithm 1 gives

$$
\begin{gathered}
F(1)=\left[\begin{array}{ccc}
-0.0872 & 0.0232 & -0.1401 \\
-0.1101 & -0.1998 & -0.0922 \\
0.0013 & 0.2260 & 0.0624 \\
-0.0313 & 0.0651 & 0.0698
\end{array}\right] \\
F(2)=\left[\begin{array}{cc}
0.0204 & -0.1834 \\
0.4108 & 0.1671 \\
-0.0018 & -0.1035 \\
-0.1475 & -0.2631
\end{array}\right]
\end{gathered}
$$

The computations required have been performed in MATLAB using the YALMIP parser [27] and the SeDuMi solver [28]. The eigenvalues of $\bar{A}(r)-\bar{B}(r) F(r) \Theta(r)$ in the first and second modes are equal to $\Lambda_{c}(1)=\{0.7701,0.2075,-0.4208,-0.2431\}$ and $\Lambda_{c}(2)=$ $\{-0.5325 \pm 0.6198 i, 0.5718,0.0624\}$, respectively. It can be checked that in each fixed mode the closed-loop system is stable along the pass.

\section{Conclusions}

The paper has developed a parametric description of the set of stabilizing controllers for discrete repetitive processes with uncertain parameters and Markovian jumps. The role of free parameters is played by the matrices resembling the weighting matrices in Letov's theory of analytic design of an optimal controller (LQR theory). This approach allows the use of efficient techniques from convex analysis to obtain LMI-based algorithms for computing the gain matrix. The feasibility of these LMIs does, however, depend on the weighting matrices in the cost function. The constructive selection of these matrices is not a simple task, and no comprehensive solution is known. The existing results are either applicable to particular cases [29], or are very difficult to use [30]. An additional 
constraint for 2D systems arises from the special block diagonal structure of the matrix in the quadratic Lyapunov function.

Further research should aim to extend all the results of this paper to other classes of $n \mathrm{D}$ systems $(n \geq 2$,). Potential application areas include iterative learning networked control and networked control of repetitive processes.

\section{Acknowledgment}

This work is supported in part by the Russian Foundation for Basic Research, project no. 10-08-00843_a.

\section{Appendix A. Proof of Theorem 4}

Necessity. Let the pair $W=\operatorname{diag}\left[W_{1} W_{2}\right], F=\left[F_{1} F_{2}\right]$ be a solution of the system of inequalities (17). Then there exist matrices $Q(\gamma)=Q(\gamma)^{T} \geq 0$ and $R(\gamma)=R(\gamma)^{T}>0$ such that

$$
\begin{aligned}
&(\bar{A}(\gamma)-\bar{B}(\gamma) F \Theta)^{T} W(\bar{A}(\gamma)-\bar{B}(\gamma) F \Theta)-W+Q(\gamma) \\
&+(F \Theta)^{T} R(\gamma) F \Theta<0, \quad \gamma \in \Gamma_{v}
\end{aligned}
$$

Rearranging (A.1) yields

$$
\begin{array}{r}
\bar{A}(\gamma)^{T} W \bar{A}(\gamma)-W+(F \Theta)^{T}\left(\bar{B}(\gamma)^{T} W \bar{B}(\gamma)+R(\gamma)\right) F \Theta \\
-(F \Theta)^{T} \bar{B}(\gamma)^{T} W \bar{A}(\gamma)-\bar{A}(\gamma)^{T} W \bar{B}(\gamma) F \Theta+Q(\gamma)<0, \gamma \in \Gamma_{v} .
\end{array}
$$

Setting

$$
K(\gamma)=F \Theta-\left(\bar{B}(\gamma)^{T} W \bar{B}(\gamma)+R(\gamma)\right)^{-1} \bar{B}(\gamma)^{T} W \bar{A}(\gamma), \gamma \in \Gamma_{v}
$$

(A.2) can be rewritten as

$$
\begin{aligned}
\bar{A}(\gamma)^{T} W \bar{A}(\gamma)-W & -\bar{A}(\gamma)^{T} W \bar{B}(\gamma)\left(\bar{B}(\gamma)^{T} W \bar{B}(\gamma)+R(\gamma)\right)^{-1} \bar{B}(\gamma)^{T} W \bar{A}(\gamma)+ \\
& +K(\gamma)^{T}\left(\bar{B}(\gamma)^{T} W \bar{B}(\gamma)+R(\gamma)\right) K(\gamma)+Q(\gamma)<0, \gamma \in \Gamma_{v}
\end{aligned}
$$

Now, introducing

$$
L(\gamma)=\left(\bar{B}(\gamma)^{T} W \bar{B}(\gamma)+R(\gamma)\right) K(\gamma), \gamma \in \Gamma_{v}
$$

and substituting (A.5) into (A.4) gives (20); (18) and (19) follow from (A.3), (A.5) and from the fact that $F \Theta$ is a constant matrix.

Sufficiency. Suppose there exist matrices $W=W^{T}$ and $F$ satisfying (18) and (20). Then the matrix $L(\gamma)$ is defined by (A.5), with $K(\gamma)$ given by (A.3). Hence,

$$
\begin{array}{r}
0>\bar{A}(\gamma)^{T} W \bar{A}(\gamma)-\bar{A}(\gamma)^{T} W \bar{B}(\gamma)\left(\bar{B}(\gamma)^{T} W \bar{B}(\gamma)+R(\gamma)\right)^{-1} \bar{B}(\gamma)^{T} W \bar{A}(\gamma) \\
+L(\gamma)^{T}\left(\bar{B}(\gamma)^{T} W \bar{B}(\gamma)+R(\gamma)\right)^{-1} L(\gamma)+Q(\gamma)-W=\left(\bar{A}(\gamma)^{T}\right. \\
-\bar{B}(\gamma) F \Theta)^{T} W\left(\bar{A}(\gamma)^{T}-\bar{B}(\gamma) F \Theta\right)-W+Q(\gamma)+(F \Theta)^{T} R(\gamma)(F \Theta) \\
\geq\left(\bar{A}(\gamma)^{T}-\bar{B}(\gamma) F \Theta\right)^{T} W\left(\bar{A}(\gamma)^{T}-\bar{B}(\gamma) F \Theta\right)-W, \gamma \in \Gamma_{v}
\end{array}
$$

It now follows from (A.6) that (17) holds and the proof is complete. 


\section{Appendix B. Proof of Theorem 5}

It follows from taking the expectation of both sides in (36) and (37) that

$$
\mathrm{E}\left[V_{1}\left(x_{k+1, p+1}, r_{k, p+1}\right)+V_{2}\left(y_{k+1, p}, r_{k+1, p}\right)\right] \leq \lambda \mathrm{E}\left[V_{1}\left(x_{k+1, p}, r_{k, p}\right)+V_{2}\left(y_{k, p}, r_{k, p}\right)\right],
$$

where $0<\lambda=\frac{c_{2}-c_{3}}{c_{2}}<1$. We have for $k$ from 0 to $N$ and $p$ from $N$ to 0 ,

$$
\begin{aligned}
\mathrm{E}\left[V_{1}\left(x_{1, N+1}, r_{0, N+1}\right)+V_{2}\left(y_{1, N}, r_{1, N}\right)\right] & \leq \lambda \mathrm{E}\left[V_{1}\left(x_{1, N}, r_{0, N}\right)+V_{2}\left(y_{0, N}, r_{0, N}\right)\right], \\
\mathrm{E}\left[V_{1}\left(x_{2, N}, r_{1, N}\right)+V_{2}\left(y_{2, N-1}, r_{2, N-1}\right)\right] & \leq \lambda \mathrm{E}\left[V_{1}\left(x_{2, N-1}, r_{1, N-1}\right)+V_{2}\left(y_{1, N-1}, r_{1, N-1}\right)\right], \\
\mathrm{E}\left[V_{1}\left(x_{3, N-1}, r_{2, N-1}\right)+V_{2}\left(y_{3, N-2}, r_{3, N-2}\right)\right] & \leq \lambda \mathrm{E}\left[V_{1}\left(x_{3, N-2}, r_{3, N-2}\right)+V_{2}\left(y_{2, N-2}, r_{2, N-2}\right)\right], \\
& \vdots \\
\mathrm{E}\left[V_{1}\left(x_{N+1,1}, r_{N, 1}\right)+V_{2}\left(y_{N+1,0}, r_{N+1,0}\right)\right] & \leq \lambda \mathrm{E}\left[V_{1}\left(x_{N+1,0}, r_{N, 0}\right)+V_{2}\left(y_{N, 0}, r_{N, 0}\right)\right] .
\end{aligned}
$$

Adding both sides of these inequalities and using the trivial identity

$$
\mathrm{E}\left[V_{1}\left(x_{N+2,0}, r_{N+1,0}\right)+V_{2}\left(y_{0, N+1}, r_{0, N+1}\right)\right]=\mathrm{E}\left[V_{1}\left(x_{N+2,0}, r_{N+1,0}\right)+V_{2}\left(y_{0, N+1}, r_{0, N+1}\right)\right]
$$

results in

$$
\begin{array}{r}
\mathrm{E}\left[\sum_{j=0}^{N+1} V_{1}\left(x_{N+2-j, j}, r_{N+1-j, j}\right)+V_{2}\left(y_{N+1-j, j}, r_{N+1-j, j}\right)\right] \leq \\
\leq \lambda \mathrm{E}\left[\sum_{j=0}^{N} V_{1}\left(x_{N+1-j, j}, r_{N-j, j}\right)+V_{2}\left(y_{N-j, j}, r_{N-j, j}\right)\right]+ \\
+\mathrm{E}\left[V_{1}\left(x_{N+2,0}, r_{N+1,0}\right)+V_{2}\left(y_{0, N+1}, r_{0, N+1}\right)\right] .
\end{array}
$$

Hence,

$$
\begin{array}{r}
\mathrm{E}\left[\sum_{j=0}^{N+1} V_{1}\left(x_{N+2-j, j}, r_{N+1-j, j}\right)+V_{2}\left(y_{N+1-j, j}, r_{N+1-j, j}\right)\right] \leq \\
\leq \mathrm{E}\left[\sum_{j=0}^{N+1} \lambda^{j}\left(V_{1}\left(x_{N+2-j, 0}, r_{N+1-j, 0}\right)+V_{2}\left(y_{0, N+1-j}, r_{0, N+1-j}\right)\right)\right],
\end{array}
$$

and using (36),

$$
\mathrm{E}\left[\sum_{j=0}^{N}\left(\left|x_{N+1-j, j}\right|^{2}+\left|y_{N-j, j}\right|^{2}\right)\right] \leq \alpha \mathrm{E}\left[\sum_{j=0}^{N} \lambda^{j}\left(\left|x_{N+1-j, 0}\right|^{2}+\left|y_{0, N-j}\right|^{2}\right)\right],
$$

where $\alpha=c_{2} / c_{1}$. The last inequality implies that

$$
\begin{array}{r}
\sum_{N=0}^{M} \mathrm{E}\left[\sum_{j=0}^{N}\left(\left|x_{N+1-j, j}\right|^{2}+\left|y_{N-j, j}\right|^{2}\right)\right] \leq \\
\leq \alpha\left[\left(1+\lambda+\ldots+\lambda^{M}\right) \mathrm{E}\left(\left|x_{1,0}\right|^{2}+\left|y_{0,0}\right|^{2}\right)+\left(1+\lambda+\ldots+\lambda^{M-1}\right) \mathrm{E}\left(\left|x_{2,0}\right|^{2}+\left|y_{0,1}\right|^{2}\right)+\right. \\
\left.+\left(1+\lambda+\ldots+\lambda^{M-2}\right) \mathrm{E}\left(\left|x_{3,0}\right|^{2}+\left|y_{0,2}\right|^{2}\right)+\ldots+\mathrm{E}\left(\left|x_{M+1,0}\right|^{2}+\left|y_{0, M}\right|^{2}\right)\right] \leq \\
\leq \alpha\left[\left(1+\lambda+\ldots+\lambda^{M}\right) \mathrm{E} \sum_{N=0}^{M}\left(\left|x_{N+1,0}\right|^{2}+\left|y_{0, N}\right|^{2}\right)\right] .
\end{array}
$$


Since (33) holds and $0<\lambda<1$, it follows that the right-hand side of this inequality is bounded as $M \rightarrow \infty$. Consequently, the series in the left-hand side of the considered inequality is convergent and

$$
\mathrm{E}\left[\sum_{j=0}^{N}\left(\left|x_{N+1-j, j}\right|^{2}+\left|y_{N-j, j}\right|^{2}\right)\right] \rightarrow 0
$$

as $N \rightarrow \infty$, and the system (30), (32) is asymptotically stable in the mean square. This concludes the proof. 


\section{References}

[1] Rogers E., Owens D. H. Stability Analysis for Linear Repetitive Processes. Lecture Notes in Control and Information Sciences, Vol. 175. Berlin: Springer-Verlag, 1992.

[2] Rogers, E., Gałkowski, K., Owens, D. H. Control Systems Theory and Applications for Linear Repetitive Processes, Lecture Notes in Control and Information Sciences, Vol. 349. Berlin: Springer-Verlag, 2007.

[3] Hladowski L., Galkowski K., Cai Z., Rogers E., Freeman C. T., Lewin P. L. Experimentally supported 2D systems based iterative learning control law design for error convergence and performance // Control Engineering Practice. 2010. V. 18. P. 339348

[4] Roberts P. D. Numerical investigations of a stability theorem arising from 2dimensional analysis of an iterative optimal control algorithm // Multidimensional Systems and Signal Processing. 2000. V. 11. P. 109-124.

[5] Dullerud G. E. and D'Andrea R. Distributed control of heterogeneous systems // IEEE Transactions on Automatic Control. 2004. V. 49. P. 2113-2128.

[6] Rabenstein R., Trautmann L. Towards a framework for continuous and discrete multidimensional systems // International Journal of Applied Mathematics and Computer Science. 2003. V. 13. P. 73-86.

[7] Cichy B., Augusta P., Rogers E.,and Gałkowski K., Hurák Z. On the control of distributed parameter systems using a multidimensional systems setting // Mechanical Systems and Signal Processing. 2008. V. 22. P. 1566-1581.

[8] Boyd S., El Ghaoui L., Feron E., Balakrishnan V., Linear Matrix Inequalities in System and Control Theory. Philadelphia: SIAM, 1994.

[9] Balandin D. V., Kogan M. M. Synthesis of Control Laws Based on Linear Matrix Inequalities. Moscow: Nauka, 2007.

[10] Letov A.M. Analytical design of controllers //I. Avtom.i Telemekh. 1960.No.4. P.436441; II. 1960. No.5. P.561-568; III.1960.No.6. P. 661-665; IV. 1961. No.4. P. 425-435; V. 1962. No.11. P. 1405-1413.

[11] Gadewadikar J., Lewis F. L., Xie L.,Kucera V., Abu-Khalaf M. Parameterization of all stabilizing $H_{\infty}$ static state-feedback gains: Application to output-feedback design // Automatica. 2007. V. 43. P. $1597-1604$.

[12] Pakshin P.V., Soloviev S.G., Peaucelle D. Parametrization of stabilizing controls in stochastic systems // Automation and Remote Control. 2009. V. 70, No. 9. P. 15141527.

[13] Pakshin P., Galkowski K., Sulikowski B., Rogers E. Parametrization Based Synthesis of Static Feedback Stabilizing Controllers for Uncertain Discrete Linear Repetitive Processes // Proceedings of the 3rd IEEE Multi-conference on Systems and Control. Saint Petersburg, Russia. July 8-10, 2009. P. 962-967. 
[14] Kazakov I.Ye., Artem'ev V.M. Optimization of Dynamical Systems with Random Structure. Moscow: Nauka, 1980.

[15] Mariton M. Jump Linear Systems in Automatic Control. New York: Marcel Dekker, 1990.

[16] Kats, I. Ya. and Martynyuk, A. A. Stability and Stabilization of Nonlinear Systems with Random Structure. London: Taylor \& Francis, 2002.

[17] Pakshin, P.V. Disctrete Systems with Random Parameters and Structure. Moscow: Fizmatlit, 1994.

[18] Costa O.L.V., Fragoso M.D., Marques R.P. Discrete-Time Markov Jump Linear Systems. New York: Springer, 2004.

[19] Costa O.L.V., Okimura R.T., Discrete-time mean variance optimal control of linear systems with Markovian jumps and multiplicative noise // International Journal of Control. 2009. V. 82. P. 256-267.

[20] Oliveira R.C.L.F., Vargas A.N., do Val J.B.R., Peres P.L.D. Robust stability, $H_{2}$ analysis and stabilization of discrete-time Markov jump linear systems with uncertain probability matrix // International Journal of Control. 2009. V. 82. P. 470-481.

[21] Gao H., Lam J., Xu S., Wang C. Stabilization and $H_{\infty}$ control of two-dimensional Markovian jump systems // IMA Journal of Mathematical Control and Information. 2004. V. 21. P. 377-392.

[22] Wu L., Shi P., Gao H., Wang C. $H_{\infty}$ filtering for 2D Markovian jump systems // Automatica. 2008. V. 44. P. 1849-1858.

[23] Roesser R.P. A discrete state-space model for linear image processing // IEEE Transactions on Automatic Control. 1975. V. AC-20. P. 1- 10.

[24] Fornasini E., Marchesini G. Doubly indexed dynamical systems: state models and structural properties // Mathematical Systems Theory, V. 12. 1978. P. 59-72.

[25] Skelton R.E.,Iwasaki T., Grigoriadis K.M. A Unified Algebraic Approach to Linear Control Design. London: Taylor \& Francis, 1997.

[26] Ait Rami M., El Ghaoui L. LMI optimization for nonstandard Riccati equation arising in stochastic control // IEEE Trans. Automat. Control. 1996. V. 41. P. 1666-1671.

[27] Löfberg J. YALMIP : A Toolbox for Modeling and Optimization in MATLAB, 2004. URL: http:// control.ee.ethz.ch/ joloef/ yalmip.php.

[28] Sturm J. F. Using SeDuMi 1.02, a MATLAB toolbox for optimization over symmetric cones // Optimization Methods and Software. 1999. V. 11-12. P. 625-653. URL: http://sedumi.mcmaster.ca/.

[29] Johnson C. D. The "unreachable poles" defect in LQR theory: analysis and remedy // Int. J. Control. 1988. V. 47. P. 697-709.

[30] Fujii T. A new approach to the LQ design from the viewpoint of the inverse regulator problem // IEEE Trans. Automat. Control. 1987. V. 32. P. 995-1004. 